\title{
Mathematical Modeling of Crosslinked Polyacrylic Based Hydrogels: Physical Properties and Drug Delivery
}

\section{Filippo Bisotti}

Politecnico di Milano

\section{Fabio Pizzetti}

Politecnico di Milano

\section{Giuseppe Storti}

Politecnico di Milano

Filippo Rossi ( $\nabla$ filippo.rossi@polimi.it)

Politecnico di Milano https://orcid.org/0000-0003-2665-120X

\section{Research Article}

Keywords: hydrogels, drug delivery, swelling dynamics, mathematical modeling, transport phenomena

Posted Date: September 1st, 2021

DOl: https://doi.org/10.21203/rs.3.rs-791682/v1

License: (c) (i) This work is licensed under a Creative Commons Attribution 4.0 International License. Read Full License 
MATHEMATICAL MODELING OF CROSSLINKED POLYACRYLIC BASED HYDROGELS: PHYSICAL PROPERTIES AND DRUG DELIVERY

Filippo Bisottia; Fabio Pizzettia ; Giuseppe Storti and Filippo Rossi*

Department of Chemistry, Materials and Chemical Engineering "Giulio Natta", Politecnico di Milano, Milan, Italy

a equally contributed

* corresponding author, email: filippo.rossi@polimi.it, Tel: (+39) 02 23993145, fax: (+39) 0223993180. 


\begin{abstract}
Recently, hydrogels have gained significant importance in different applications, such as tissue engineering and drug delivery. They are 3D-structures of hydrophilic polymers held together through physical or chemical cross-linking. Important is their ability to swell in presence of solvents, forming elastic gels able to mantain their original shape. Furthermore, this scaffolds slowly degrade in the physiological environment, leading the growing tissue to replace the former filled site. In this work, hydrogels have been synthetized using branched polyacrilic acid (Carbomer) cross-linked with an aliphatic polyetherdiamine (elastamine). In particular, we focused on the description of their equilibrium conditions in swollen state and the dynamic simulation of the swelling process. These hydrogels exhibited a peculiar swelling behaviour characterized by an overshoot of the volume increase before reaching the equilibrium. Notably, such behavior was found at different $\mathrm{pH}$ values. In this manuscript, the swelling behavior was studied by mathematical modelling. Moreover, the ability of these devices to release drugs was also examined through a literature model to understand the different operating transport mechanisms.
\end{abstract}

Keywords: hydrogels, drug delivery, swelling dynamics, mathematical modeling, transport phenomena. 


\section{Graphical abstract}

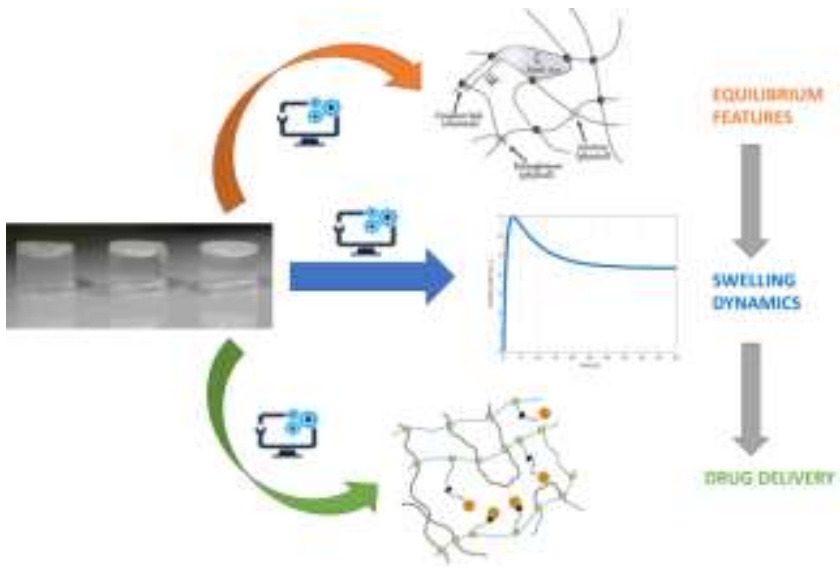




\section{Introduction}

Hydrogels are biomedical devices that gained increasing interest as potential drug delivery carriers for advanced injury treatments [1]. These applications are possible due to their peculiar structure and physical properties [2]. Hydrogels are 3D-structures made of polymer chains interconnected by a crosslinker: the backbone structure as well as the extent of crosslinking determine their mechanical and swelling properties [3,4]. Indeed, hydrogels absorb a huge amount of solvent, entrapping liquid and increasing their volume even by many times [5]. If swelling is carried out in presence of a drug, the drug is released when the swollen hydrogel is put in a different medium. Hence, swelling and drug delivery are intimately interconnected [6]. Liquid absorption reaches equilibrium conditions once the tensional forces on the polymer chains are balanced by elastic forces. Therefore, mechanical properties strongly affect the swelling behaviour and resulting drug delivery performances [7].

The main parameters characterizing swollen hydrogels are mesh size, crosslinking density, average molecular weight between two following crosslinks and overall volume. The swelling kinetics describes the dynamic evolution in time of the liquid solvent uptake within the hydrogel structure [8]. Usually, the swelling dynamics is described by the evaluation of the swelling ratio $Q_{V}$, defined as:

$$
\mathrm{Q}_{\mathrm{V}}=\frac{\mathrm{W}_{\mathrm{s}}-\mathrm{W}_{\mathrm{d}}}{\mathrm{W}_{\mathrm{d}}}
$$

where $\mathrm{W}_{\mathrm{s}}$ and $\mathrm{W}_{\mathrm{d}}$ are the weights of the swollen and dry hydrogel respectively. In the literature, two possible swelling dynamics have been experimentally verified: (i) the monotonously increasing approach to equilibrium swelling, which is the most common, and (ii) the "overshoot swelling" which involves intermediate non-equilibrium configurations [9]. The latter dynamic trend is quite uncommon and exhibited by a limited set of hydrogels.

Focusing on drug delivery, as mentioned before, load bearing capacity is one of the pivotal properties of hydrogels. In biological applications, a drug or other therapeutic molecules can be first loaded inside such structure and then released to an external medium [10]. Drug delivery systems (DDS) are structures able to encapsulate drugs and then release them in a controlled way. In recent years, hydrogels have been used as controlled DDS, since they enable the maintenance of the drug concentration inside the therapeutic range, thus avoiding the risk of under and overdosing [11]. Not only the drug release mechanism is important for the description of the release kinetics, but also the degradation properties, since usually the overall release behavior is a combination of diffusion mechanism and degradation (that can be bulk or by surface erosion) [12].

Moving to modeling considerations, it is not surprising that the mathematical modelling of hydrogels is attracting increasing interest since it enables their design and customization according to target applications and specifications. The physical properties of the swollen hydrogels at equilibrium, swelling dynamics, and drug delivery properties can be 
predicted [13]. Moreover, modelling can be applied in designing hydrogel devices aimed to drug delivery, since drug released amount, releasing rate, and discharging time can be also predicted. However, in both cases such predictions rely on experimental tests, given the complex interplay between swelling dynamics and hydrogel features [14].

In this work, the potential of mathematical modelling to determine physical properties of swollen hydrogels and interpret the swelling dynamics will be assessed, with emphasis on the interpretation of non-equilibrium states. Hydrogel samples made of Carbomer (polyacrylic acid) as main constituent and elastamine RE-2000 as crosslinker will be examined [15]. Moreover, a stochastic method will be applied for characterizing the drug delivery of Rhodamine B from the same hydrogel samples. In this last section, it will be proven that it is possible to apply simplified models and extend the proposed approach also to over-swollen hydrogels. 


\section{Experimental part}

The experimental data used in this work have been previously published by Mauri et al. [15], and the experimental setup and procedures are only briefly summarized in the following.

Hydrogel synthesis - Carbomer has been dissolved into $1.5 \mathrm{~mL}$ of distilled water, while elastamine RE-2000 in $8.5 \mathrm{~mL}$. Different proportions have been tested, as reported in Table 1. Using a syringe, the two solutions have been mixed and a given volume has been inserted into steel cylinders (diameter $1.1 \mathrm{~cm}$ ) and refrigerated at $-20^{\circ} \mathrm{C}$ for 8 hours. At this point, a physical gel is already formed. Before promoting the transition from physical to chemical crosslinking, the prepared hydrogels have been lyophilized. Such transition occurs after 25-30 min in a microwave oven (maximum admissible temperature of $\left.80^{\circ} \mathrm{C}\right)$.

\section{Table 1}

Swelling experiments - Swelling tests have been performed by weighting the dried chemically crosslinked gels and soaking them in a known volume of distilled water. Different pHs have been tested, controlling them using a buffer solution as aqueous medium. At fixed timepoints, the swollen hydrogels have been weighted and the swelling ratios have been evaluated.

Drug release experiments - In these tests, rhodamine $\mathrm{B}(\mathrm{RhB})$ has been chosen as drug mimetics. The drug loading has been obtained by soaking the dried gels into a solution of RhB in distilled water. After equilibrium has been reached, the supernatant has been withdrawn and analyzed through UV-Vis at the excitation wavelength of RhB in water, exploiting the Lambert-Beer law [16], the quantity of absorbed drug has been estimated. Then, the loaded hydrogels have been immersed in a given volume of water (or buffer solution, PBS for physiological conditions). Release assays have been performed by withdrawing a known volume of supernatant and replacing it with fresh distilled water, to maintain the concentration gradient. The withdrawn solution has then been analyzed by UV-Vis and the final percentage of release estimated. 


\section{Modeling part}

\section{Equilibrium swelling}

Before analyzing the swelling dynamics, the swelling behaviour at equilibrium is discussed. The two key parameters for describing the hydrogel properties at equilibrium are mesh size $(\xi)$ and crosslinking density $(v)$. The theoretical model used has been derived from the Flory-Rehner theory [17] under the following simplifying assumptions:

- $\quad$ The number average molecular weight of the backbone $\left(\mathrm{M}_{\mathrm{n}}\right)$ is very large;

- $\quad$ The volume fraction of polymer before swelling is equal to unity, since the sample underwent lyophilization.

Accordingly, the Flory-Rehner equation becomes:

$$
\mathrm{M}_{\mathrm{c}}=\frac{\frac{\Omega_{1}}{\bar{v}}\left(\Phi_{2, \mathrm{~s}}^{1 / 3}-\frac{\Phi_{2, \mathrm{~s}}}{2}\right)}{\ln \left(1-\Phi_{2, \mathrm{~s}}\right)+\Phi_{2, \mathrm{~s}}+\chi \Phi_{2, \mathrm{~s}}^{2}}
$$

where $\bar{v}$ is the specific volume of the backbone polymer, $\Omega_{1}$ the molar volume of the swelling medium, $\Phi_{2, \mathrm{~s}}$ the polymer volume fraction after swelling, $\chi$ the Flory polymer-solvent interaction factor and $\mathrm{M}_{\mathrm{c}}$ the average molecular weight between crosslinks.

The mesh size $\xi$ can then be evaluated given the end-to-end distance of the solvent-free state $r_{0}$, in turn estimated by Eqn. (3) $[18,19]$ :

$$
r_{0}=C_{n}^{1 / 2} l\left(\frac{2 M_{c}}{M_{r}}\right)^{1 / 2}
$$

where $C_{n}$ is the characteristic Flory ratio (available in the literature for many polymers), and l the characteristic monomer length of the bond along the polymer backbone (i.e., C-C bond). Once this distance is known, $\xi$ and $v$ can be evaluated through Eqn. (4) and (5) [18,20], respectively:

$$
\begin{gathered}
\xi=\mathrm{r}_{0} \Phi_{2, \mathrm{~s}}^{1 / 3} \\
v=\frac{\rho_{\mathrm{p}}}{\mathrm{M}_{\mathrm{c}}}
\end{gathered}
$$

\section{Swelling dynamics}

Carbomer shows a completely different swelling dynamics with respect to most of the hydrogels previously analyzed in the literature. Namely, instead of the classical sigmoidal curve, an overshoot is observed before reaching the equilibrium condition. This can be referred to as "overshooting effect". The actual presence and the intensity of such overshoot is a function of different parameters, such as crosslinking degree, temperature, ionic strength and $\mathrm{pH}$ of the solution, and the release of unreacted polymer during swelling. The model used in this work to describe the swelling dynamics has been developed in previous works by Diez-Peña et al. [21,22]. According to NMR experimental results, they proposed a model 
able to consider different "types" of water with different mobilities depending on the soaking $\mathrm{pH}$ medium. The same authors applied this model to N-iPPAm-co-MAA copolymers, which exhibited overshooting effect during the swelling process. Given the similar functional groups (i.e., similar interactions between the functionalities and the sorrounding medium) on the backbone chains and the similar swelling behaviour, the same approach has been adopted here for Carbomer-based hydrogels. According to the model, water molecules are classified into different classes and a kinetic scheme has been proposed to describe the transformation of the different kinds of water within the system. The water molecules classification is slightly modified to fit our system:

1. Initially, the functional groups (carboxylic moieties for the Carbomer hydrogel) are unbounded leading to a nonstiff structure enabling water uptake by instantaneous diffusion inside the system. Water absorbtion starts and the overall water amount that the hydrogel is able to store is denoted as $A_{1}$.

2. Water molecules continously diffuse inside the gel causing its volume growth. In this phase the water is continuously absorbed and distributed within the crosslinked structure. This type of water is referred as $A_{2}$ and the corresponding hydrogel structure is named "primary structure". It should be noted that $\mathrm{A}_{2}$ is a "product" of the water initially uptaken $\left(A_{1}\right)$, while its initial amount is negligible.

3. While swelling goes on, the medium $\mathrm{pH}$ plays a significant role since it promotes the rearrangement of the hydrogel structure through hydrogen bonding and/or electrostatic interactions. Indeed, the carboxylic moieties suitably appear in the either protonated or deprotonated form according to the medium $\mathrm{pH}$. The resulting internal interactions lead to a more compact and rigid structure called "secondary structure" and the successive shrinkage. The structure stiffening is responsible for the overswelling response: the entrapped water inside the over-swollen hydrogel is named $\mathrm{A}_{3}$.

4. Finally, the stiffening process leads to a structure shrinkage since the water content is higher than the equilibrium value. Part of the previously entrapped water $\left(A_{3}\right)$ is released, and this quantity will be referred to as $A_{4}$.

Given this mechanistic picture, the following kinetic scheme involving the "transformation" of water types has been proposed.

$$
A_{1} \underset{k_{2}}{\stackrel{k_{1}}{\rightleftarrows}} A_{2} \underset{k_{4}}{\stackrel{k_{3}}{\rightleftarrows}} A_{3} \underset{k_{6}}{\stackrel{k_{5}}{\rightleftarrows}} A_{4}
$$

Six different kinetic constants are involved, one for each step in the kinetic scheme. However, as highlighted in the previous cited works, since the initial water uptake is driven by fast water absorption and diffusion, the reverse reactions (water release and water back diffusion) are negligible. Hence, the first two kinetic steps can be considered as irreversible. The scheme can be simplified as follows, with a single reversible step: 


$$
A_{1} \stackrel{k_{1}}{\longrightarrow} A_{2} \stackrel{k_{3}}{\longrightarrow} A_{3} \underset{k_{6}}{\stackrel{k_{5}}{\rightleftarrows}} A_{4}
$$

The dynamics of the different types of water has been described through the following set of differential equations:

$$
\begin{gathered}
\frac{\mathrm{dA}_{1}}{\mathrm{dt}}=-\mathrm{k}_{1} \mathrm{~A}_{1} \\
\frac{\mathrm{dA}_{2}}{\mathrm{dt}}=\mathrm{k}_{1} \mathrm{~A}_{1}-\mathrm{k}_{3} \mathrm{~A}_{2} \\
\frac{\mathrm{dA}_{3}}{\mathrm{dt}}=\mathrm{k}_{3} \mathrm{~A}_{2}-\mathrm{k}_{5} \mathrm{~A}_{3}+\mathrm{k}_{6} \mathrm{~A}_{4} \\
\frac{\mathrm{dA}_{4}}{\mathrm{dt}}=\mathrm{k}_{5} \mathrm{~A}_{3}-\mathrm{k}_{6} \mathrm{~A}_{4}
\end{gathered}
$$

where $\mathrm{k}_{1}$ is the rate constant of the transformation of $A_{1}$ into $A_{2}, k_{3}$ the one of the second irreversible reaction (overswelling state), and $\mathrm{k}_{5}$ and $\mathrm{k}_{6}$ are those of water uptake and excess water release, respectively. The swelling ratio evaluated on a mass basis is defined as:

$$
\mathrm{Q}_{\mathrm{t}}=\frac{\mathrm{m}_{\mathrm{t}}-\mathrm{m}_{0}}{\mathrm{~m}_{0}}=\frac{\mathrm{m}_{\mathrm{w}}}{\mathrm{m}_{0}}
$$

where $\mathrm{m}_{0}$ is the initial mass, $\mathrm{m}_{\mathrm{t}}$ the mass at time $\mathrm{t}$ and $\mathrm{m}_{\mathrm{w}}$ the total amount of water entrapped inside the hydrogel at the same time. The last quantity is the sum of the water in the primary and secondary structure, that is $A_{2}$ and $A_{3}$. As soon as the swelling equilibrium is established, the swelling ratio is equal to the value of $A_{3}$. Taking advantage of the analytical solution of the previous set of differential equations, the following expression is worked out:

$$
\begin{gathered}
\mathrm{Q}_{\mathrm{t}}=\left[\frac{\mathrm{k}_{3}}{\mathrm{k}_{3}-\mathrm{k}_{1}} \frac{\mathrm{k}_{1}-\mathrm{k}_{6}}{\mathrm{k}_{1}-\mathrm{k}_{5}-\mathrm{k}_{6}} \mathrm{e}^{-\mathrm{k}_{1} \mathrm{t}}+\frac{\mathrm{k}_{1}}{\mathrm{k}_{1}-\mathrm{k}_{3}}\left(\mathrm{e}^{-\mathrm{k}_{3} \mathrm{t}}-\mathrm{e}^{-\mathrm{k}_{1} \mathrm{t}}\right)-\frac{\mathrm{k}_{1}}{\mathrm{k}_{1}-\mathrm{k}_{3}} \frac{\mathrm{k}_{3}-\mathrm{k}_{6}}{\mathrm{k}_{3}-\mathrm{k}_{5}-\mathrm{k}_{6}} \mathrm{e}^{-\mathrm{k}_{3} \mathrm{t}}\right. \\
\left.+\frac{\mathrm{k}_{1}}{\mathrm{k}_{1}-\mathrm{k}_{5}-\mathrm{k}_{6}} \frac{\mathrm{k}_{5}}{\mathrm{k}_{5}+\mathrm{k}_{6}} \frac{\mathrm{k}_{3}}{\mathrm{k}_{3}-\mathrm{k}_{5}-\mathrm{k}_{6}} \mathrm{e}^{-\left(\mathrm{k}_{5}+\mathrm{k}_{6}\right) \mathrm{t}}+\frac{\mathrm{k}_{6}}{\mathrm{k}_{6}+\mathrm{k}_{5}}\right] \mathrm{a}_{0}
\end{gathered}
$$

where $\mathrm{a}_{0}$ is the initial amount of water inside the hydrogel. At equilibrium (very long times), the last equation reduces to:

$$
\mathrm{Q}_{\mathrm{t}}(t=\infty)=\mathrm{Q}_{\mathrm{eq}}=\frac{\mathrm{k}_{6}}{\mathrm{k}_{6}+\mathrm{k}_{5}} \mathrm{a}_{0}
$$

Eqn. (11) and (12) involve five adjustable parameters: the four kinetic constants and the initial water content.

\section{Drug delivery}

Two different regimes have been taken into consideration when dealing with drug release, the operative regime and the so-called anomalous one.

As far as the operative regime is concerned, the drug diffusion coefficient can be evaluated from the Stokes-Einstein equation [23]. The effective diffusivity inside the hydrogel matrix can thus be evaluated using a correlation proposed by Lustig and Peppas [24]: 


$$
\frac{\mathcal{D}_{\mathrm{g}}}{\mathcal{D}_{0}}=\left(1-\frac{\mathrm{r}_{\mathrm{s}}}{\xi}\right) \exp \left(-\mathrm{Y} \frac{\Phi}{1-\Phi}\right)
$$

where $\mathcal{D}_{0}$ is the diffusion coefficient at infinite dilution (i.e., the one evaluated with the Stokes-Einstein equation), $\mathrm{r}_{\mathrm{s}}$ the hydrodynamic diameter of the solute, $\xi$ the hydrogel mesh size, $\Phi$ the polymer volumetric fraction in the swollen hydrogel and $\mathrm{Y}$ the ratio of the critical volume required for a successful translational movement of the solute molecule and the average free volume per molecule of liquid. According to Lin et al. [25], Y can be assumed equal to unity without loss of accuracy. Once the diffusion coefficient has been evaluated, and given relaxation time and characteristic length, it is possible to evaluate the Deborah number (De), defined as the ratio between relaxation and diffusion characteristic times [25-27]. If Deborah number is smaller than 1 the rate determining step is diffusion, while the drug release is governed by the swelling process when Deborah number is larger than 1.

Moving to the anomalous diffusion regime, different models have been proposed in the literature $[25,27,28]$. For istance, a general approach has been developed by Korsmeyer and Peppas [29-31] using the following power law fitting function:

$$
\frac{\mathrm{m}(\mathrm{t})}{\mathrm{m}_{\mathrm{eq}}}=\mathrm{k} \cdot \mathrm{t}^{\mathrm{n}}
$$

where $\mathrm{m}(\mathrm{t})$ is the mass of drug released at a specific time and $\mathrm{m}_{\mathrm{eq}}$ is the total released mass when equilibrium conditions is reached. Despite its simplicity, this approach is effective to predict the intial drug release profile (i.e., until $\mathrm{m}(\mathrm{t}) / \mathrm{m}_{\mathrm{eq}}<$ 0.60). On the other hand, it fails systematically to describe experimental observations when drug delivery is approaching equilibrium conditions, as it will be verified in the next section. This limitation may be explained considering the lack of physical meaning of the adjustable parameters appearing in its formulation.

For different hydrogel geometris, Liu and Metters [25] demonstrated the existence of lower and upper limits of exponent $\mathrm{n}$ and associated such threshold values to the corresponding drug release regime (i.e., diffusion- or swelling-controlled release process; see Table 2). For n values falling within these boundaries, the so-called "abnormal" drug diffusion takes place, corresponding to a combination of the two limiting behaviors, diffusion- and swelling-controlled. In the present work, the hydrogel samples are rassemble as cylindrical elements.

\section{Table 2}

In this work, the model proposed by Kosmidis et al. [32-34] has been selected. Such model is based on the Weibull function:

$$
\frac{\mathrm{m}(\mathrm{t})}{\mathrm{m}_{\mathrm{eq}}}=1-\exp \left(-\mathrm{at} \mathrm{t}^{\mathrm{b}}\right)
$$

As emphasized by the same authors, a clear physical meaning has been assigned to each parameter appearing in Eq(15) by Monte Carlo simulations. Parameter a is directly related to the probability of the drug molecule to escape from the solid matrix, whereas the geometrical coefficient b reflects the specific surface per unit of volume $\left(\mathrm{m}^{2} / \mathrm{m}^{3}\right)$. As expected, 
the larger is the specific surface, the higher is the probability of drug escape from the hydrogel. Differently from the power-law approach, this method enables to properly represent the profile of the entire release curve, as will be proven in the next section.

\section{Results and Discussion}

Equilibrium swelling

The numerical values of the main parameters estimated for the different hydrogels are summarized in Table 3 .

\section{Table 3}

To check the consistency of the estimated swelling parameters, these results have been compared to those reported in the literature as evaluated by the same equilibrium model for a similar carbomer [35]. All the parameter values exhibit the same order of magnitude and this finding supports the reliability of the applied approach.

Focusing on crosslinking density, largely different values are estimated for the same sample depending on the used external medium. In particular, at neutral $\mathrm{pH}$ each hydrogel is weaker and less compact than at higher or lower $\mathrm{pH}$, with variations up to one order of magnitude. Since the chemical crosslinking is constant for all carbomer samples, an additional "force" is operating able to intensify the interactions among the polymer chains and making the structure stiffer. This increase of stiffness is reflected by an increase of "effective" crosslinking density, where the term effective is used to remember it accounts for both chemical and physical crosslinking. The physical crosslinking is the key of this phenomenon: its effect overlaps the one of the chemical crosslinking or, even better, its contribute prevails forming strong physical interconnections among the polymer chains that effectively supports and toughens the matrix. This consideration will become clearer when discussing the experimental results of swelling dynamics.

As a final remark, some authors (e.g., [18]), taking advantage of the theoretical framework previously developed by Schaefer [36], have investigated the relation between mesh size and polymer fraction. The simplest proposed model is the power-law relationship reported below:

$$
\xi=\alpha+\beta \cdot \phi^{\mathrm{n}}
$$

Despite its simplicity, this power-law works properly in predicting the evolution of the mesh size with the polymer volume fraction for $\mathrm{n}=-0.50$ and $\phi<0.10$ regardless of the solvent and polymer nature. Our results further support this capability and indicate that the validity range of the model can be slightly enlarged, as well proven by the results in Fig. 1 and Table 4.

\section{Table 4}

Fig. 1 


\section{Swelling dynamics}

Experimental data have been taken from the work of Mauri et al. [15]. Note that, even though the overall experimental time was $350 \mathrm{~h}$, the time scale in the figure is limited to $80 \mathrm{~h}$ in order to better visualize the overshoots. The five adjustable parameters of the swelling model have been estimated by fitting the experimental data at different conditions, namely different carbomer to elastamine ratios and different $\mathrm{pH}$ values. The latter values have been set from basic $(\mathrm{pH}=3)$ to neutral $(\mathrm{pH}=7)$ and to acidic conditions $(\mathrm{pH}=10)$ using a buffer solution to maintain constant the solution $\mathrm{pH}$ to the specific value all along each experiment. The specific concentrations, along with the names of the different samples, have already been reported in Table 1 . The experimental data are well described by the calculated curves whose refitted parameters are collected in Table 5. The refitted values are similar to those proposed in the literature [21,37]; in particular, the order of magnitude of each parameter and the corresponding ratios are preserved. The largest discrepancy compared to the literature results is present in the parameters $\mathrm{k}_{1}$ and $\mathrm{k}_{3}$. On the other hand, the specific hydrogel formulation (polymer to crosslinker ratio) and the different buffer solution $\mathrm{pH}$ adopted in the several experimental campaigns may justify this difference. Indeed, in the light of the effect of the solvent in the interaction among polymer chains and its impact on the final hydrogel features, it is possible to conclude that these parameters may deeply affect the initial kinetic steps (water absorption and water distribution rate identified with $\mathrm{k}_{1}$ and $\mathrm{k}_{3}$ respectively) where the contact between solvent molecules and backbone structure is unavoidable.

Furthermore, by analyzing the experimental and model results in Fig. 2 it can be noticed that:

- In acidic and basic environment, the hydrogel structure exhibits a sort of intrinsic stiffness, related to the formation of hydrogen bonds at low $\mathrm{pH}$ and of electrostatic repulsive forces at high $\mathrm{pH}$. From the experimental data, it seems that the hydrogen bonds stabilize the structure more effectively than the electrostatic interactions, even though both chemical and physical crosslinks cooperate to hydrogel shrinking. Structure stiffening is possible thanks to either the protonated form of the carboxylic groups, responsible for hydrogen bonds at low to medium $\mathrm{pH}$, or the deprotonated form, which actives electrostatic forces.

- At neutral $\mathrm{pH}$, both types of physical crosslinking are weak, hence swelling dynamics is more pronounced. At these conditions, both hydrogen bonds and electrostatic forces are operative, but at much smaller extent compared to the previous cases. As soon as the overshoot is reached, the elastic contribution of swelling starts to play the dominant role, shrinking the structure and reducing the achievable equilibrium value. In fact, such value is larger than the one established at low or high $\mathrm{pH}$.

- Crosslinking density plays a key role in determining the swelling equilibrium and its effect is more evident at $\mathrm{pH}$ near to neutrality. Looking at the time evolution of $\mathrm{Q}_{\mathrm{t}}$, the higher the crosslinking density, the lower the swelling, 
both in terms of peak width and equilibrium plateau. Such effects become less relevant in acid and basic conditions, where physical entanglement exceeds the chemical ones.

- Increasing the chemical crosslinking, the impact of the physical one on swelling equilibrium decreases.

Fig. 2

A sensitivity analysis enables to deduce the impact of the different model parameters:

- $\quad \mathrm{k}_{1}$ controls the swelling-time derivative at the origin; furthermore, it affects the curvature near the maximum. This is consistent with the reported kinetic scheme, since the first reaction involves the initial uptake of water. The larger is the initial water uptake, the higher is the swelling rate (i.e., the initial slope).

- $\quad$ The ratio between $\mathrm{k}_{1}$ and $\mathrm{k}_{3}$ influences the width of the overshoot, while the value of $\mathrm{k}_{3}$ determines the peak position. Again, this is consistent with the model, since the second step in the kinetic scheme accounts for the diffusion of the solvent into the solid matrix. In case of slow rate, the overswelling peak is reached at longer time.

- $\quad \mathrm{k}_{5}$ and $\mathrm{k}_{6}$ influence the height of the final plateau, as expected according to the previous considerations on swelling equilibrium.

- $\quad \mathrm{a}_{0}$ regulates the width of the peak with respect to unswollen conditions, since the initial water content affects swelling dynamics and overshoot extent.

- $\quad \mathrm{t}$ peak the time needed to reach the peak.

\section{Table 5}

Fig. 3

Fig. 3 shows how the ratio between the first two rate parameters is function of $\mathrm{pH}$ and of the formulation used in the hydrogel production. The crosslinking density plays a relevant role at $\mathrm{pH}$ close to neutrality, while the differences between the different formulations are negligible at acidic and basic $\mathrm{pH}$. It is important to remind the physical meaning of these two parameters: while $\mathrm{k}_{1}$ determines the initial slope of the swelling curve, $\mathrm{k}_{3}$ the specific time at which the overshoot occurs.

Analyzing the time at which the maximum is reached (last column in Table 5), the following considerations can be deduced:

- In acidic environment, highly crosslinked samples swell slowly. This trend agrees with the results of Diez-Peña et al. [22], where longer times to equilibrium conditions were also found for highly crosslinked hydrogels. This behaviour is most probably due to the stronger interactions that "protect" the hydrogen-bounded complexes from breakage. This can also be due to a higher stability of hydrogen bonds themselves that prevents water diffusion into the samples. As a matter 
of fact, the maximum swelling ratios of highly crosslinked hydrogels (1-3 and 1-4) are smaller than those of slightly crosslinked ones.

- $\quad$ At pH close to neutrality, a larger difference between samples 2-2 and 1-2 compared to that between 1-3 and 14 can be observed. This behaviour supports the existence of a critical value of crosslinking extent, even though additional experiments should be done to identify such threshold value.

- At neutral pH, swelling is controlled by the chemical crosslinking. Hydrogels 2-2 and 1-2 show larger swelling compared to samples 1-3 and 1-4, as confirmed by the peak times. The stiffer hydrogels retain less water and the time needed to overcome the maximum is shorter. The opposite occurs for weaker hydrogels.

- In basic environment, the times at which the peak is reached are almost the same. In fact, under such conditions the hydrogels are held together mainly through electrostatic interactions caused by deprotonation of the carboxylic groups. The ratio polyacrylic acid/crosslinker is different in the four samples; since no carboxylic groups are present in the crosslinker, the quantity of carboxyl groups is almost the same for every sample because the amount of carbomer is constant.

Let us now consider the role of the last two kinetic constants, $\mathrm{k}_{5}$ and $\mathrm{k}_{6}$. Their values determine the second portion of the swelling curve, where the final equilibrium is established. In very acidic or basic media, independently upon the chosen formulation, the hydrogel is quite stiff and water absorption is extremely slow. On the other hand, at pH values close to neutrality the structural properties are weaker and the swelling/deswelling rates are larger. These behaviors are reflected by the estimated values of $\mathrm{k}_{5}$ and $\mathrm{k}_{6}$, as reported in Fig. 4. Furthermore, the ratio between the two kinetic constants remains almost equal at each value of $\mathrm{pH}$ and crosslinking density, meaning that, the ratio between the rates of release and recovery of water is similar independently from the hydrogel formulation. However, the external environment $\mathrm{pH}$ affects the nominal velocity of water release and uptake. At neutral $\mathrm{pH}$ the structure is more flexible, hence, these kinetic steps rates are larger compared to other anylised $\mathrm{pH}$ conditions where hydrogels present more rigid structures which hinder the release-uptake kinetic.

\section{Fig. 4}

Finally, some considerations on the structural properties of the hydrogels are helpful to describe the impact of the initial water content $\mathrm{a}_{0}$ on the model predictions. Water diffusion and crosslinking density are strongly dependent from each other, and several papers are available in the literature discussing water diffusion inside hydrogels [2]. In general, the diffusion coefficients inside the hydrogel drop at increasing crosslinking density, since the number of vacancies available for diffusion decreases. Accordingly, the hydrogels entrap less solvent at low and high $\mathrm{pH}$ values. On the contrary, at $\mathrm{pH}$ values close to neutrality, swelling is much more relevant, and the chemical crosslinking density controls the amount of water able to diffuse into the hydrogel at the beginning of the experiment: the more crosslinked the hydrogel, the lower its capability to absorb water. The estimated values of $\mathrm{a}_{0}$ shown in Fig. 5 reflect the previous arguments. 
Fig. 5

\section{Drug Delivery}

Concerning drug delivery, the model based on the Weibull function previously mentioned has been compared with a classical power-law model proposed by Peppas and coworkers [29,30]. Also in this case, the experimental values have been taken from Mauri et al. [15]. Two different Rhodamine B (RhB) loadings have been taken into consideration: the first one obtained by loading the drug inside the hydrogel matrix by diffusion, while the second one exploits the formation of an ester bond, thus chemically linking the drug to the hydrogel matrix. Results showing the comparison between the different models (Weibull and power-law refit) are shown in Fig. 6.

Fig. 6

As already mentioned, the power-law approach properly describes the behaviour of the drug release at the beginning, until $\frac{\mathrm{m}(\mathrm{t})}{\mathrm{m}_{\mathrm{eq}}} \leq 0.6 \div 0.7$. Beyond this treshold value, the predictions of the power law model become unreliable, while those of the model based on the Weibull curve agree well with the complete experimental profile. Moreover, as shown in Fig. 6, all the Weibull functions fit carefully the experimental data close to the origin as well as to equilibrium conditions, while discrepancies appear in the central region of the plots. Such discrepancies may be due to the rearrangement of the inner hydrogel structure which affects the swelling behaviour and the operative diffusion regime.

Following the approach proposed by Papadopoulou et al. [34], a relation between the parameters $\mathrm{n}$ and $\mathrm{b}$ (estimated through the power law and the Weibull curve, respectively) exists. According to our results, the $\mathrm{n}$ values agree with the predictions based on Deborah number: the diffusion is governed by a non-Fickian regime together with other phenomena, such as the swelling of the polymeric matrix and the solvent penetration into the network. The main differences arise when considering the $\mathrm{b}$ parameters. As reported in [33], a linear relation between exponent $\mathrm{n}$ and $\mathrm{b}$ parameters has been found out. In Table 6 and Table 7, the b values estimated using this linear interpolation are reported (column "regression"). Looking at the ratio between regressed and fitted values of the parameter $b$, it ranges from 1.41 to 3.33 , with an average value equal to 1.99. Regressed values about twice the fitted ones can be explained considering that Kosmidis et al. applied their model using the Higuchi law, which assumes negligible swelling during the drug release process. This is not the case for carbomer hydrogels that exhibit pronounced swelling as shown in the swelling dynamic results discussion. Therefore, in case of swellable hydrogels, the relation between $\mathrm{n}$ and $\mathrm{b}$ proposed by Kosmidis et al. still applies but asks for some correction. Namely, the same linear interpolation can be proposed in the case under examination but the coefficients are doubled:

$$
\mathrm{b}=0.7038 \cdot \mathrm{n}+0.5500
$$

Table 6 


\section{Table 7}

\section{Conclusions}

In this work, focusing on hydrogels made of carbomer and elastamine, swelling and drug release behaviors have been analyzed.

In the first case, it has been demonstrated that these hydrogels are strongly $\mathrm{pH}$ sensitive. Their swelling dynamics have been well described through a semiempirical, literature model involving five adjustable parameters. The same model is also effectively predicting the overshoot of swelling, a phenomenon rarely encountered working with hydrogels. The cause of the overshooting has been identified as the interplay between physical and chemical crosslinks, with the extent of the first ones strongly dependent upon the medium $\mathrm{pH}$.

Such understanding is of pivotal importance when dealing with drug delivery in these devices, due to the combination between non-Fickian diffusion and relaxation of the polymer matrix. The Weibull curve has been found to be a fitting function effective to describe the experimental data. On the other hand, the estimated values of the model parameters do not agree with those reported in previous literature works. Even though the numerical values are about half of those expected, still the selected fitting procedure remains successfully applicable to the highly swelling hydrogels examined in this work.

\section{Acknowledgments}

Authors would like to acknowledge Dr. Emanuele Mauri for fruitful discussion.

\section{Author contributions}

Fabio Pizzetti, Filippo Bisotti: Investigation, Methodology; First draft writing; Giuseppe Storti: Conceptualization, Data curation, Validation, Supervision, Writing-review \& editing. Filippo Rossi: Conceptualization, Writing-review \& editing, Supervision, Project administration, Funding acquisition.

\section{Funding}

Private funding

\section{Declarations}

\section{Ethical approval}

Not applicable 


\section{Consent to partecipate}

Not applicable

\section{Consent for publication}

The author transfers to Springer the non- exclusive publication rights and the warrants.

\section{Conflict of interest}

The authors declared no conflicts of interests 


\section{References}

1. Aswathy SH, Narendrakumar U, Manjubala I. Commercial hydrogels for biomedical applications. Heliyon [Internet]. Elsevier Ltd; 2020;6:e03719. Available from: https://doi.org/10.1016/j.heliyon.2020.e03719

2. Caló E, Khutoryanskiy V V. Biomedical applications of hydrogels: A review of patents and commercial products. Eur Polym J. 2015;65:252-67.

3. Chai Q, Jiao Y, Yu X. Hydrogels for Biomedical Applications: Their Characteristics and the Mechanisms behind Them. Gels. 2017;3:6.

4. Yu Shrike Zhang1 AK. Adnabces in Engineering Hydrogels. Physiol Behav. 2019;176:139-48.

5. Yahia Lh. History and Applications of Hydrogels. J Biomed Sci. 2015;04:1-23.

6. Liechty WB, Kryscio DR, Slaughter B V., Peppas NA. Polymers for drug delivery systems. Annu Rev Chem Biomol Eng. 2010;1:149-73.

7. Budianto E, Amalia A. Swelling behavior and mechanical properties of Chitosan-Poly(N-vinyl-pyrrolidone) hydrogels. J Polym Eng [Internet]. 2020;40:551-60. Available from: https://doi.org/10.1515/polyeng-2019-0169

8. Kipcak AS, Ismail O, Doymaz I, Piskin S. Modeling and Investigation of the Swelling Kinetics of AcrylamideSodium Acrylate Hydrogel. J Chem. 2014;2014.

9. Koetting MC, Peters JT, Steichen SD, Peppas NA. Stimulus-responsive hydrogels: Theory, modern advances, and applications. Mater Sci Eng R Reports. 2015;93:1-49.

10. Trucillo P. Drug Carriers: Classification, Administration, Release Profiles, and Industrial Approach. Processes. $2021 ; 9$.

11. Vigata M, Meinert C, Hutmacher DW, Bock N. Hydrogels as drug delivery systems: A review of current characterization and evaluation techniques. Pharmaceutics. 2020;12:1-45.

12. Fu, Yao; Kao WJ. Drug Release Kinetics and Transport Mechanisms of Non- degradable and Degradable Polymeric Delivery Systems. Bone. 2011;72:132-5.

13. Kowalski G, Kijowska K, Witczak M, Kuterasiński L, Lukasiewicz M. Synthesis and effect of structure on swelling properties of hydrogels based on high methylated pectin and acrylic polymers. Polymers (Basel). 2019;11:1-16.

14. Karoyo AH, Wilson LD. A review on the design and hydration properties of natural polymer-based hydrogels. Materials (Basel). 2021;14:1-36.

15. Mauri E, Naso D, Rossetti A, Borghi E, Ottaviano E, Griffini G, et al. Design of polymer-based antimicrobial hydrogels through physico-chemical transition. Mater Sci Eng C [Internet]. Elsevier; 2019;103:109791. Available from: https://doi.org/10.1016/j.msec.2019.109791

16. Wypych G, editor. 2 - PHOTOPHYSICS. Handb UV Degrad Stab (Second Ed [Internet]. Second Edi. ChemTec 
Publishing; 2015. p. 9-35. Available from: https://www.sciencedirect.com/science/article/pii/B9781895198867500048

17. Bruck SD. Extension of the Flory-Rehner theory of swelling to an anisotropic polymer system. J Res Natl Bur Stand Sect A Phys Chem. 1961;65A:485.

18. Canal T, Peppas NA. Correlation between mesh size and equilibrium degree of swelling of polymeric networks. J Biomed Mater Res. 1989;23:1183-93.

19. Caccavo D, Cascone S, Lamberti G, Barba AA. Hydrogels: Experimental characterization and mathematical modelling of their mechanical and diffusive behaviour. Chem Soc Rev. Royal Society of Chemistry; 2018;47:2357-73.

20. Slaughter B V., Khurshid SS, Fisher OZ, Khademhosseini A, Peppas NA. Hydrogels in regenerative medicine. Adv Mater. 2009;21:3307-29.

21. Díez-Peńa E, Quijada-Garrido I, Barrales-Rienda JM. Analysis of the swelling dynamics of cross-linked P(NiPAAm-co-MAA) copolymers and their homopolymers under acidic medium. A kinetics interpretation of the overshooting effect. Macromolecules. 2003;36:2475-83.

22. Díez-Peña E, Quijada-Garrido I, Barrales-Rienda JM, Schnell I, Spiess HW. Advanced 1H solid-state NMR spectroscopy on hydrogels, 1: The effect of hydrogen bonding in the collapse of poly(methacrylic acid) (PMAA) hydrogels. Macromol Chem Phys. 2004;205:430-7.

23. Miyamoto S, Shimono K. Molecular Modeling to Estimate the Diffusion Coefficients of Drugs and Other Small Molecules. Molecules. 2020;25.

24. Lustig SR, Peppas NA. Solute diffusion in swollen membranes. IX. Scaling laws for solute diffusion in gels. J Appl Polym Sci [Internet]. 1988;36:735-47. Available from: https://onlinelibrary.wiley.com/doi/abs/10.1002/app.1988.070360401

25. Lin C-C, Metters AT. Hydrogels in controlled release formulations: network design and mathematical modeling. Adv Drug Deliv Rev. Netherlands; 2006;58:1379-408.

26. Lee PI. Kinetics of drug release from hydrogel matrices. J Control Release [Internet]. 1985;2:277-88. Available from: https://www.sciencedirect.com/science/article/pii/0168365985900513

27. Grassi M, Grassi G. Mathematical modelling and controlled drug delivery: matrix systems. Curr Drug Deliv. United Arab Emirates; 2005;2:97-116.

28. Kim SW, Bae YH, Okano T. Hydrogels: Swelling, Drug Loading, and Release. Pharm Res [Internet]. 1992;9:28390. Available from: https://doi.org/10.1023/A:1015887213431

29. Peppas NA, Bures P, Leobandung W, Ichikawa H. Hydrogels in pharmaceutical formulations. Eur J Pharm Biopharm Off J Arbeitsgemeinschaft fur Pharm Verfahrenstechnik eV. Netherlands; 2000;50:27-46.

30. Peppas NA, Khare AR. Preparation, structure and diffusional behavior of hydrogels in controlled release. Adv Drug 
Deliv Rev [Internet]. 1993;11:1-35. Available from:

https://www.sciencedirect.com/science/article/pii/0169409X9390025Y

31. Ritger PL, Peppas NA. A simple equation for description of solute release II. Fickian and anomalous release from swellable devices. J Control Release [Internet]. 1987;5:37-42. Available from:

https://www.sciencedirect.com/science/article/pii/0168365987900356

32. Kosmidis K, Macheras P. Monte Carlo simulations of drug release from matrices with periodic layers of high and low diffusivity. Int J Pharm [Internet]. 2008;354:111 - 116. Available from:

https://doi.org/10.1016/j.ijpharm.2007.10.036

33. Kosmidis K, Argyrakis P, Macheras P. A Reappraisal of Drug Release Laws Using Monte Carlo Simulations: The Prevalence of the Weibull Function. Pharm Res [Internet]. 2003;20:988-95. Available from:

https://doi.org/10.1023/A:1024497920145

34. Papadopoulou V, Kosmidis K, Vlachou M, Macheras P. On the use of the Weibull function for the discernment of drug release mechanisms. Int J Pharm. Netherlands; 2006;309:44-50.

35. Yang Z, Peng H, Wang W, Liu T. Crystallization behavior of poly( $\varepsilon$-caprolactone)/layered double hydroxide nanocomposites. J Appl Polym Sci. 2010;116:2658-67.

36. Schaefer DW. A unified model for the structure of polymers in semidilute solution. Polymer (Guildf) [Internet]. 1984;25:387-94. Available from: https://www.sciencedirect.com/science/article/pii/0032386184902921

37. Yin Y, Ji X, Dong H, Ying Y, Zheng H. Study of the swelling dynamics with overshooting effect of hydrogels based on sodium alginate-g-acrylic acid. Carbohydr Polym. 2008;71:682-9. 


\section{Table captions}

Table 1 - Formulations of all the samples studied in this work

Table 2 - Values of exponent $\mathrm{n}$ and drug release regime (diffusion controlled or swelling controlled) for different geometries according to $\mathrm{Eq}(14)$.

Table 3 - Parameter values for swelling equilibrium estimated from experiments

Table 4 - Parameters in mesh size fitting

Table 5 - Numerical values of the fitting parameters at different $\mathrm{pH}$ medium for carbomer samples

Table 6 - Relation between the fitting parameter $\mathrm{b}$ and $\mathrm{n}$ for $\mathrm{RhB}$ release

Table 7 - Relation between the fitting parameter $\mathrm{b}$ and $\mathrm{n}$ for Esther-RhB release

\section{Figure captions}

Fig. 1 - Fitting curves (solid lines) and estimated mesh sizes (dots) for the different carbomer hydrogels. Vertical dashed line stands for the upper limit in polymer volume fraction as suggested in [29]

Fig. 2 - Effect of pH on swelling dynamics of all the samples: carbomer 2-2 (A), 1-2 (B), 1-3 (C) and 1-4 (D). Curves are model results, symbols experimental data. Different colors identify the $\mathrm{pH}$ values: red $=3$, green $=7$, blue $=10$

Fig. 3 - Effect of $\mathrm{pH}$ on the $\mathrm{k}_{1} / \mathrm{k}_{3}$ ratio for each sample: 2-2 (red), 1-2 (green), 1-3 (blue), 1-4 (yellow)

Fig. 4 - Effect of $\mathrm{pH}$ on the estimated values of the kinetic constants of swelling $\left(\mathrm{k}_{5}\right)$ and deswelling $\left(\mathrm{k}_{6}\right)$

Fig. 5 - Effect of pH on the initial water content ( $\mathrm{a}_{0}$ ) for all samples: hydrogel 2-2 (red), 1-2 (green), 1-3 (blue) and 1-4 (yellow)

Fig. 6 - Comparison of RhB (1) and Esther-RhB (2) release for hydrogel 2-2 (blue) and 1-2 (red) at $\mathrm{pH}=3(\mathrm{~A}), \mathrm{pH}=7$ (B) and $\mathrm{pH}=10(\mathrm{C})$ using Weibull refitting function (solid lines) as in Kosmidis and coworkers [15,32-34] and powerlaw (dashed lines) as in Peppas and coworkers [29-31] 
Figures

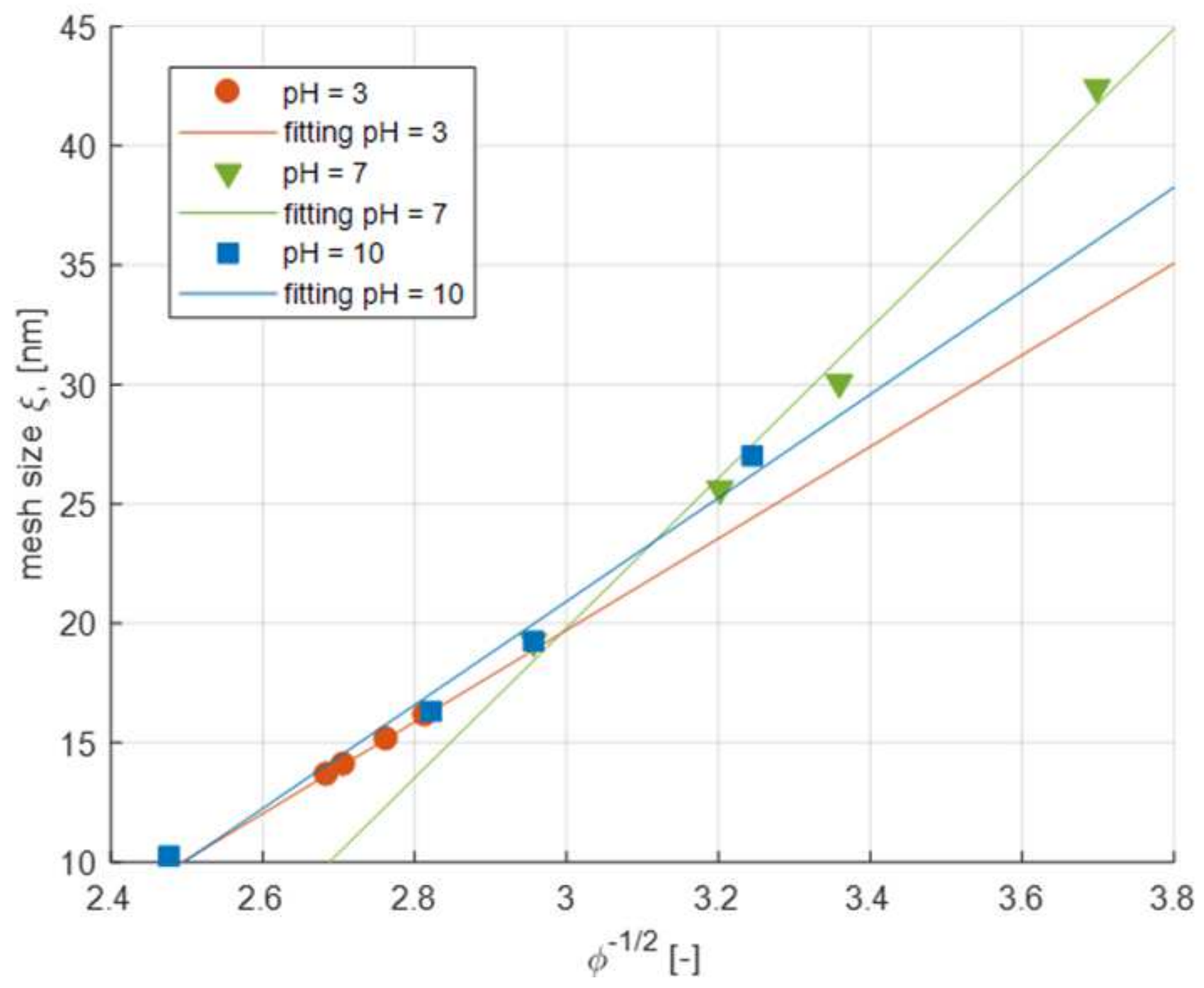

Figure 1

Fitting curves (solid lines) and estimated mesh sizes (dots) for the different carbomer hydrogels. Vertical dashed line stands for the upper limit in polymer volume fraction as suggested in [29] 

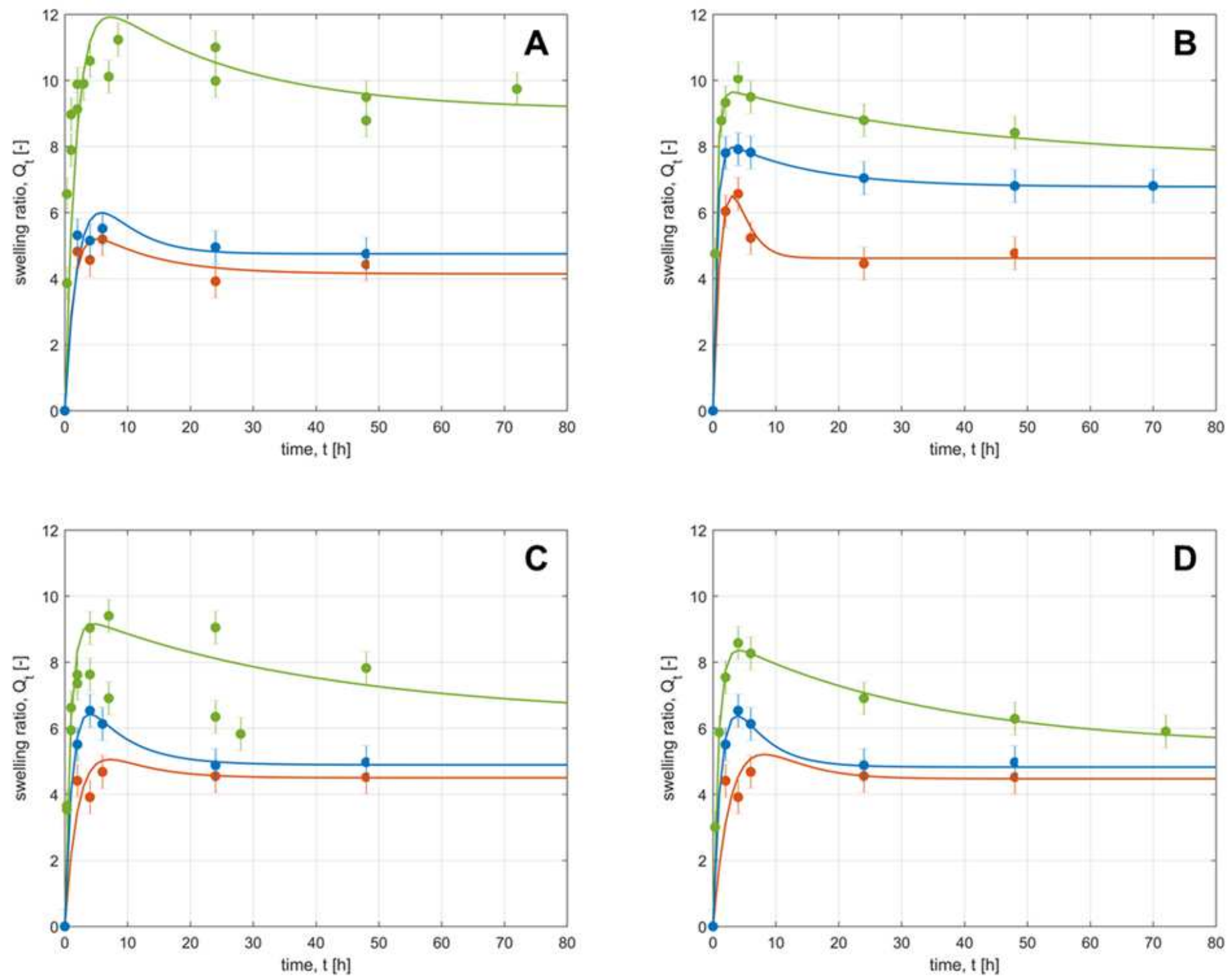

Figure 2

Effect of pH on swelling dynamics of all the samples: carbomer 2-2 (A), 1-2 (B), 1-3 (C) and 1-4 (D). Curves are model results, symbols experimental data. Different colors identify the $\mathrm{pH}$ values: $\mathrm{red}=3$, green $=7$, blue $=10$ 


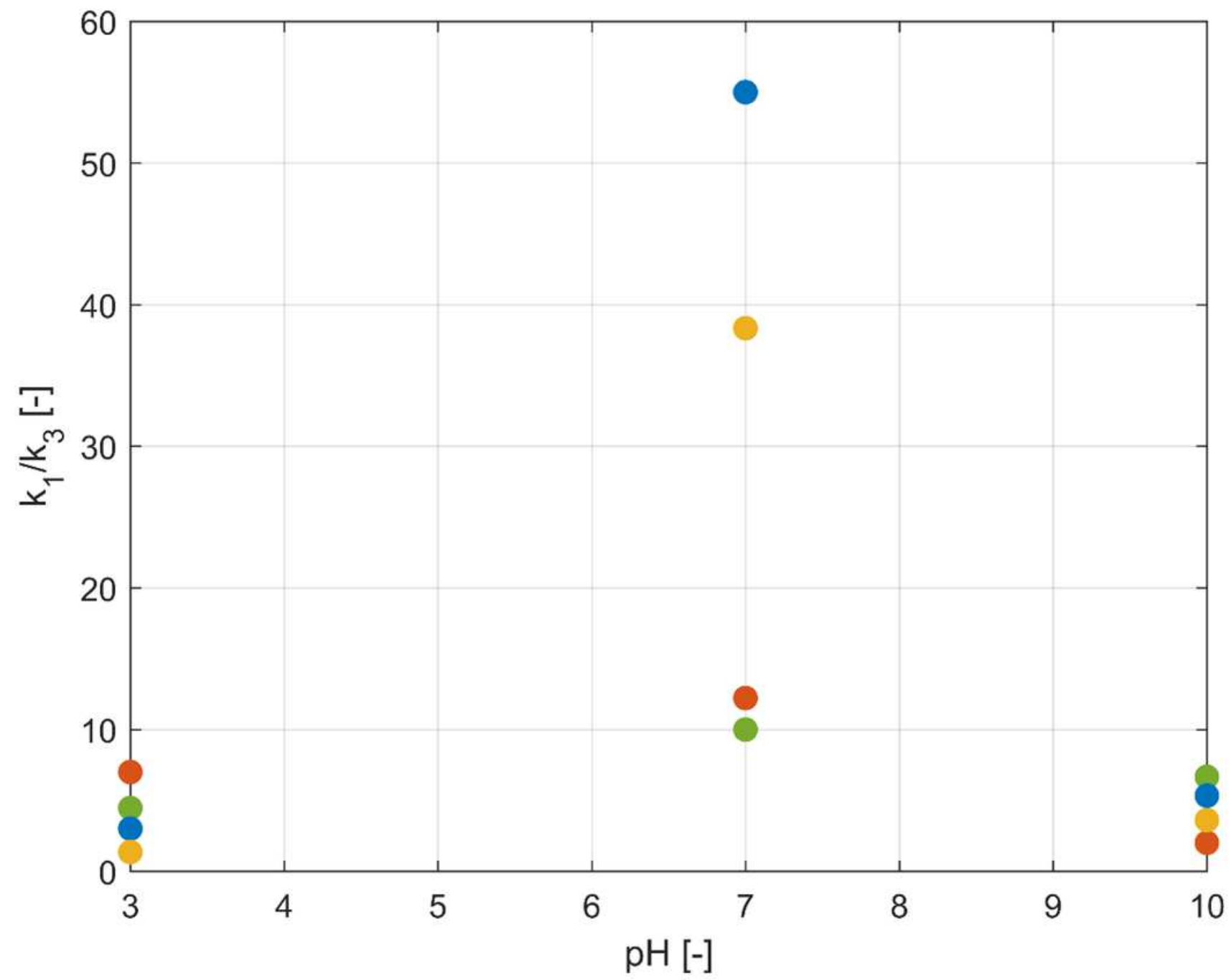

Figure 3

Effect of $\mathrm{pH}$ on the $\mathrm{k}_{-}{ }^{1 / \mathrm{k} \_3}$ ratio for each sample: 2-2 (red), 1-2 (green), 1-3 (blue), 1-4 (yellow) 

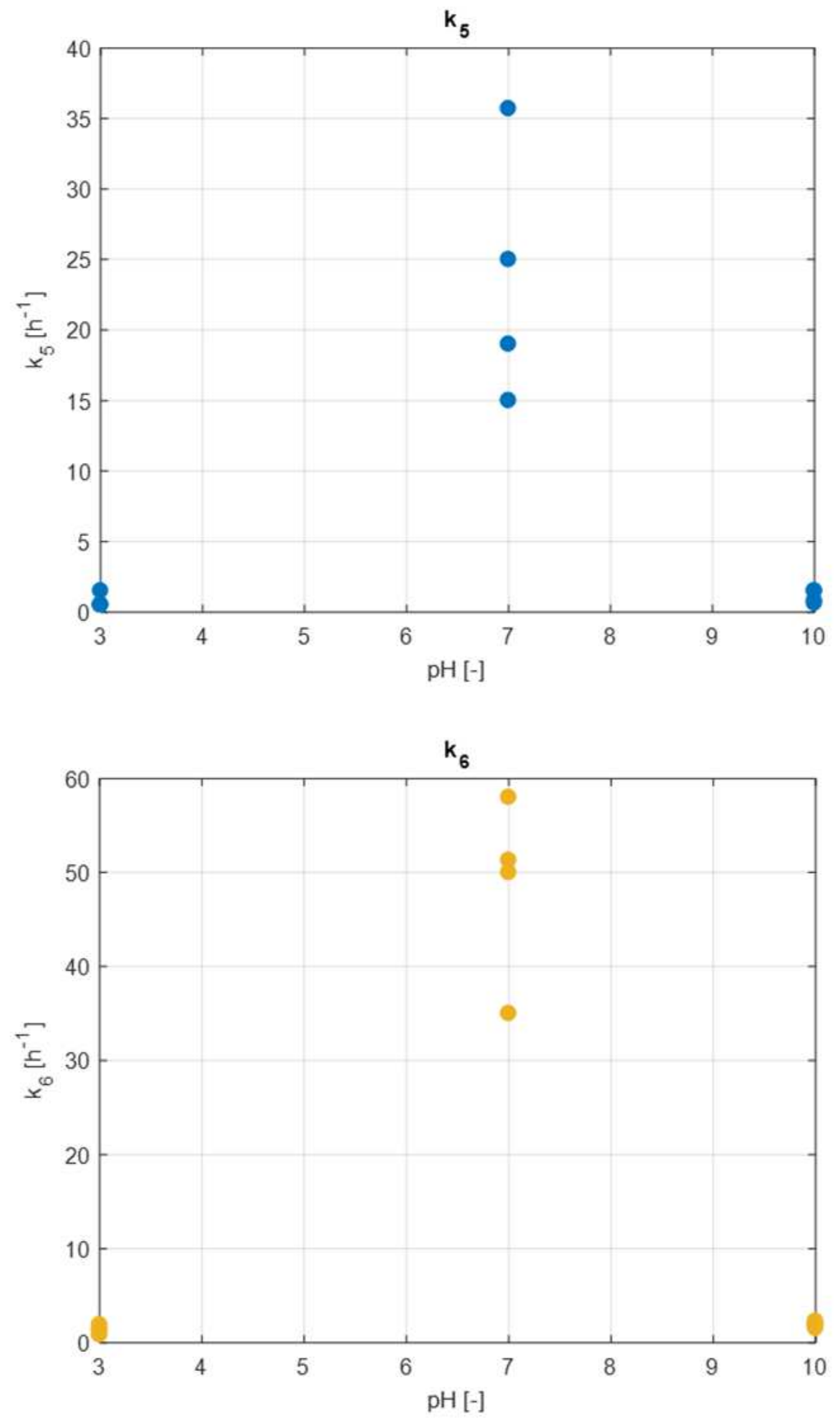

Figure 4

Effect of $\mathrm{pH}$ on the estimated values of the kinetic constants of swelling (k5) and deswelling (k6) 


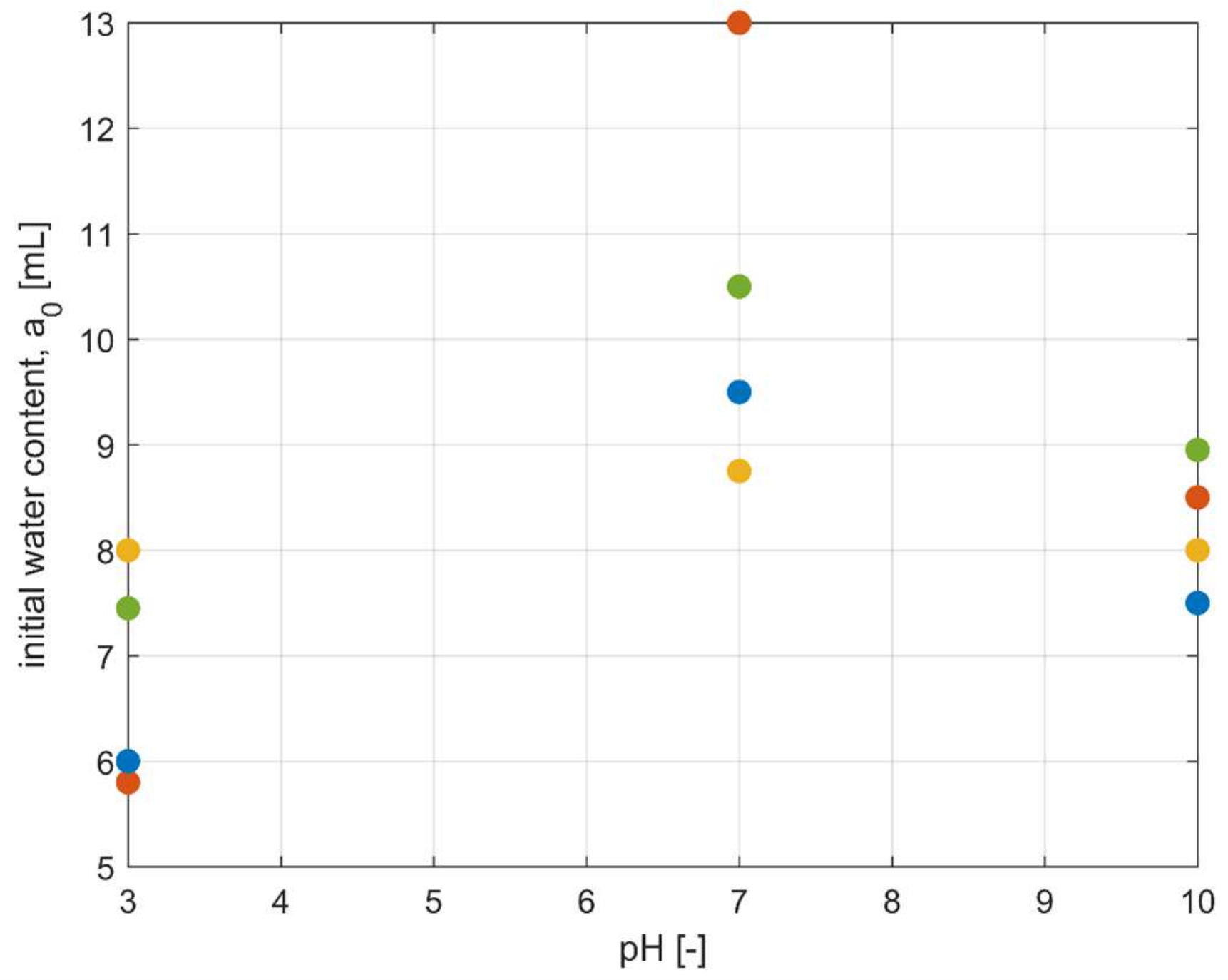

Figure 5

Effect of pH on the initial water content (a0) for all samples: hydrogel 2-2 (red), 1-2 (green), 1-3 (blue) and 1-4 (yellow) 

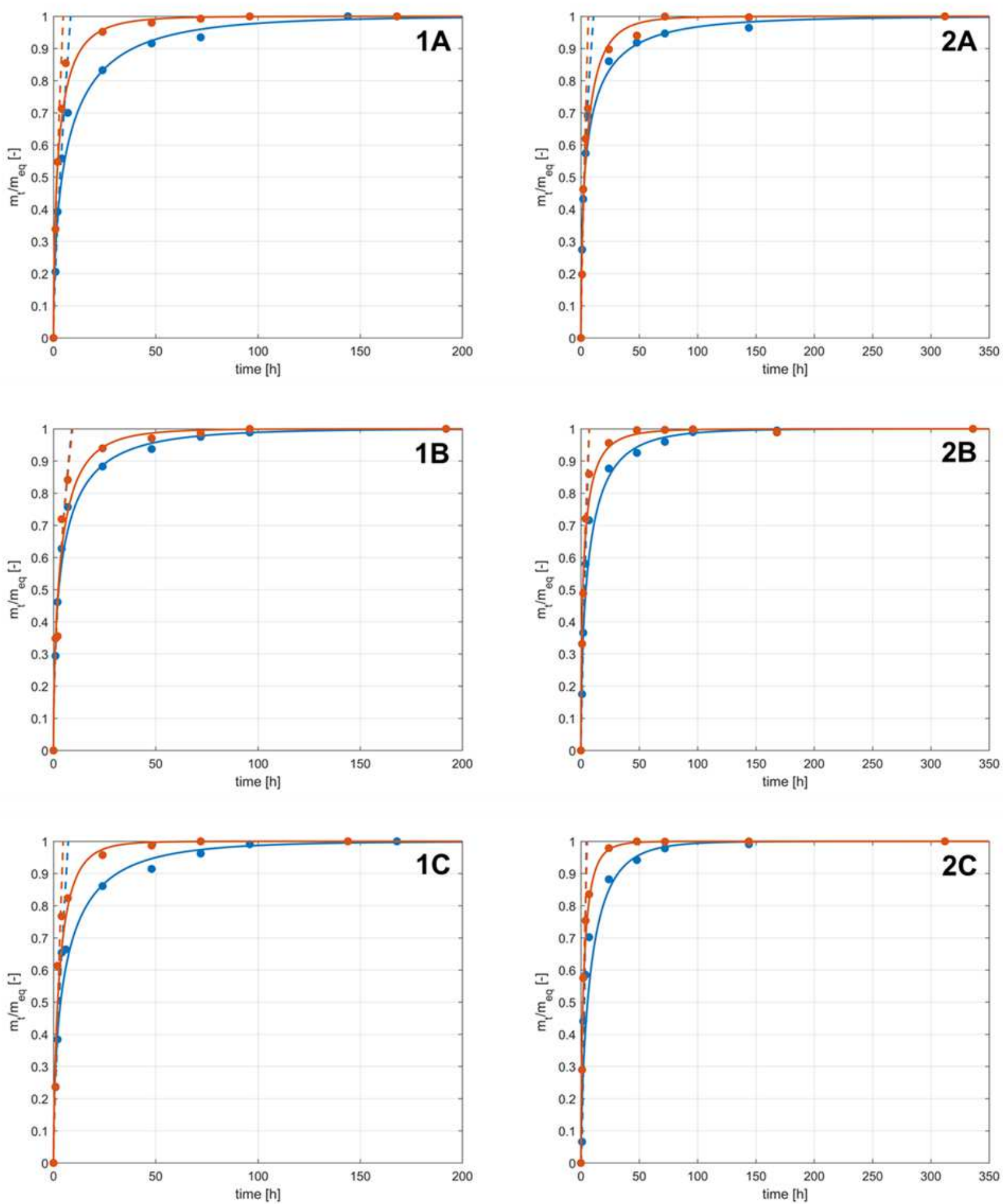

Figure 6

Comparison of $\mathrm{RhB}(1)$ and Esther-RhB (2) release for hydrogel 2-2 (blue) and 1-2 (red) at pH = $3(\mathrm{~A}), \mathrm{pH}=$ 7 (B) and $\mathrm{pH}=10$ (C) using Weibull refitting function (solid lines) as in Kosmidis and coworkers [15,3234] and power-law (dashed lines) as in Peppas and coworkers [29-31]

\section{Supplementary Files}


This is a list of supplementary files associated with this preprint. Click to download.

- graphicalabstract.png

- supporting.docx 